\title{
Adjuvant Chuna manual therapy in subjects with cervicogenic dizziness (CHERIE study): a study protocol for a prospective, pragmatic, assessor-blind, randomized controlled trial
}

Shin S, Kim J, Yu A, Seo H, Shin M, Hong S, Jung C, Kim K, Cho J, Yoo J, Lee E

College of Korean Medicine, Kyung Hee University, Seoul, Republic of Korea

\section{Introduction}

Cervicogenic dizziness is dizziness triggered by movement or positioning of the cervical spine and is often accompanied by neck pain or stiffness. A few clinical studies have explored the effects of Chuna Manual Therapy (CMT) on cervicogenic dizziness, but most have been of low quality.

\section{Purpose}

This is a prospective, pragmatic, assessor-blind, randomized controlled trial aimed at testing the efficacy and safety of adjuvant CMT over 6 weeks in patients (20-70 years old) with cervicogenic dizziness, under usual care treatments.

\section{Method}

Forty patients with cervicogenic dizziness will be randomly allocated to CMT or usual care (UC) groups, in a 1:1 ratio (Figure 1). Extensive screening procedures, including examinations for central nervous system problems and nystagmus, will be applied to exclude other dizziness-inducing disorders. The eligible participants will receive 12 sessions of CMT plus UC or only UC over 6 weeks. CMT includes mandatory and discretionary techniques (Table 1), whereas UC includes electrotherapy, thermotherapy, and patient education. The efficacy will be evaluated primarily as the change in Dizziness Handicap Inventory scores across 6 weeks. The severity and frequency of dizziness, the level of neck pain or stiffness, and the cervical range of motion, will be evaluated using validated tools. Changes in the stress level and quality of life will also be explored. Safety will be assessed by adverse events analysis (Table 2). The data will be statistically analyzed, and $P$ values below 0.05 will be deemed significant.

\section{Results}

The study protocol and the informed consent form have been peer-reviewed and approved by the Institutional Review Board of Kyung Hee University Korean Medicine Hospital on 11 August 2017 (KOMCIRB-170717-HR-026). And the trial has been registered on ClinicalTrials.gov (NCT03291912. Registered 19 September 2017, https://clinicaltrials.gov/show/NCT03291912).

\section{Conclusions}

This is expected to be the first randomized controlled study in Korea to apply CMT for cervicogenic dizziness.

\section{Competing interests}

The authors declare no conflict of interests.

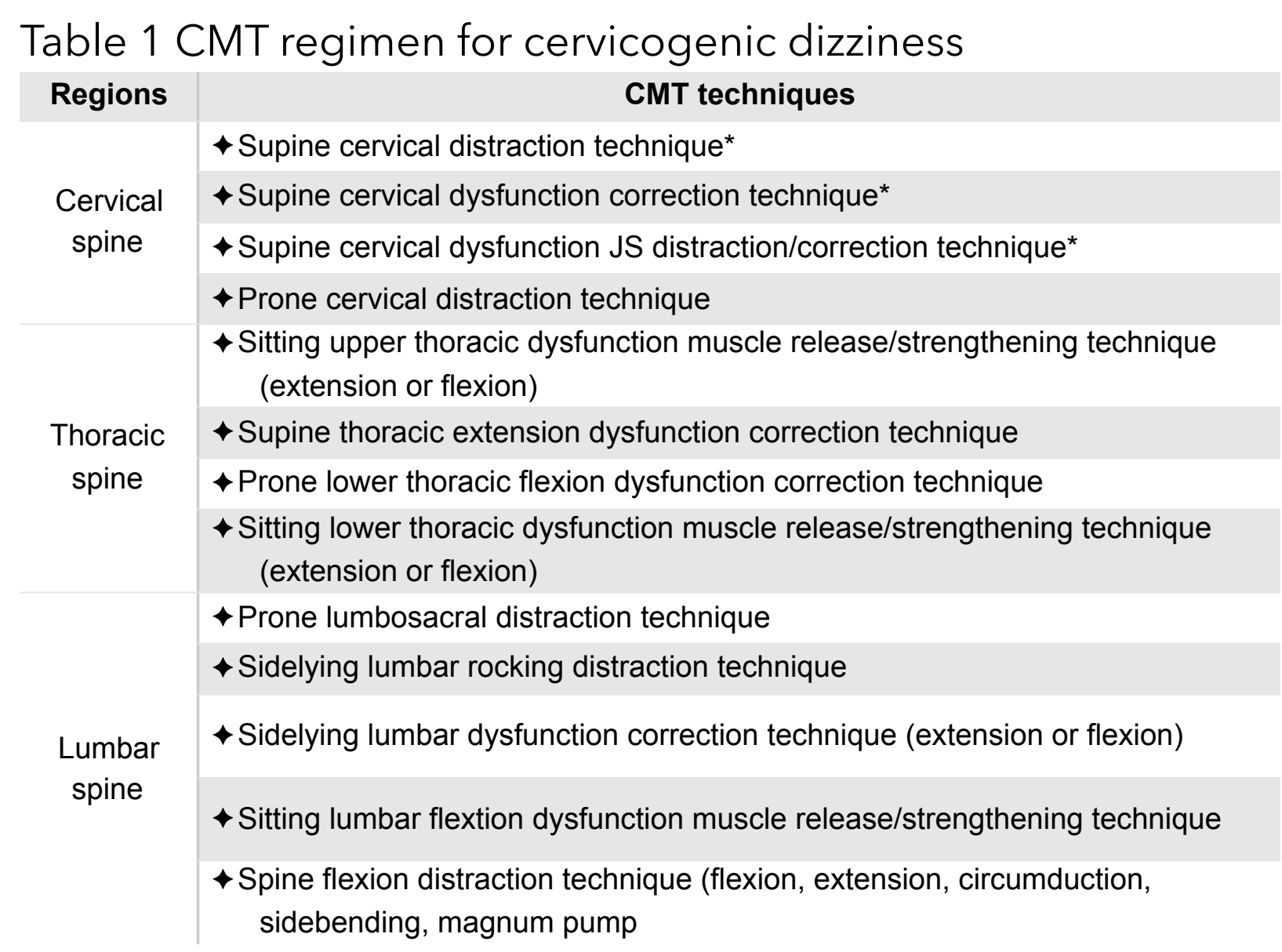

Note The techniques marked by asterisks are mandatory, whereas the others are discretionary. The practitioners will evaluate each patient before the CMT sessions and perform individually-necessary techniques to improve cervicogenic dizziness.

Table 2 Study timetable

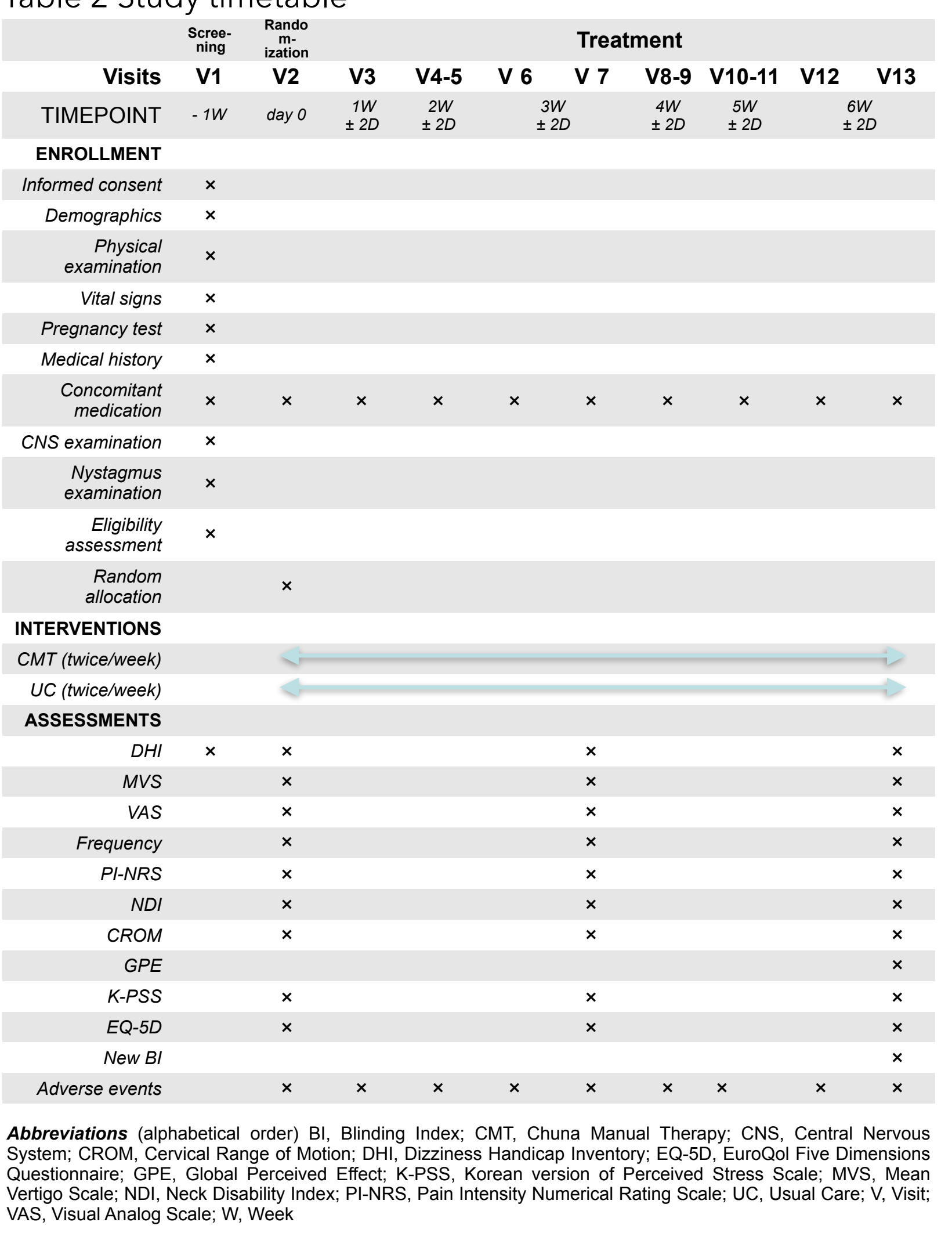

